\title{
Correlación entre la resonancia magnética y la ecocardiografía transtorácica en la evaluación de la insuficiencia pulmonar en pacientes pediátricos con cardiopatía congénita
}

\author{
Perla Roxana Aguilar-Segura ${ }^{\mathrm{a}}$, Horacio Márquez-González ${ }^{\mathrm{b}, *}$, \\ Sandra Patricia Antúnez-Sánchez ${ }^{\mathrm{c}}$, Lucelli Yáñez-Gutiérrez ${ }^{\mathrm{b}}$, \\ María de J. Estrada-Loeza ${ }^{\mathrm{d}}$ y Gabriela Meléndez-Ramírez ${ }^{\mathrm{e}}$
}

\author{
a Hospital de Ginecología y Pediatría Número 7, Cancún, Quintana Roo. Instituto Mexicano del Seguro Social, Cancún, \\ Quintana Roo, México \\ b Servicio de Cardiopatías Congénitas, Hospital de Cardiología, Centro Médico Nacional Siglo XXI, Instituto Mexicano del Seguro \\ Social, Distrito Federal, México \\ ' Cardiología Pediátrica y Ecocardiografía. Centro Médico Nacional 20 de Noviembre, Instituto de Seguridad y Servicios Sociales \\ de los Trabajadores del Estado, Distrito Federal, México \\ ¿ Servicio de Cardiología pediátrica. Hospital de Pediatría, Centro Médico Nacional Siglo XXI, Distrito Federal, México \\ e Resonancia Magnética, Instituto Nacional de Cardiología "Ignacio Chávez", Distrito Federal, México
}

Recibido el 13 de julio de 2015; aceptado el 27 de octubre de 2015

Disponible en Internet el 1 de febrero de 2016

\section{PALABRAS CLAVE \\ Defectos cardiacos congénitos; \\ Ecocardiografía; \\ Imagen por \\ resonancia magnética}

\begin{abstract}
Resumen
Objetivo: Establecer la correlación y la concordancia del grado de severidad de insuficiencia pulmonar entre la ecocardiografía y la resonancia magnética en la población pediátrica de un hospital de tercer nivel de atención.

Métodos: Se estudiaron 20 pacientes con diagnóstico de cardiopatía congénita con insuficiencia pulmonar, a quienes se les realizaron ecocardiograma transtorácico y resonancia magnética. Se calculó: relación de la vena contracta y el chorro de insuficiencia pulmonar, tiempo de hemipresión, presencia de flujo diastólico reverso en tronco pulmonar e índice de insuficiencia pulmonar; en cuanto a la resonancia magnética: la fracción y el volumen regurgitante. Se realizó análisis de correlación mediante coeficiente de correlación de Spearman y prueba de Tau B. Resultados: La tetralogía de Fallot corregida representó el $60 \%$ de la muestra. La correlación fue significativa en la fracción y el volumen regurgitante de la resonancia magnética, así como en las variables ecocardiográficas índice de insuficiencia pulmonar en modo $M$, vena contracta y porcentaje de vena contracta/arteria pulmonar. La correlación entre los grados de insuficiencia pulmonar por resonancia magnética y ecocardiografía fue $0,85(p<0,001)$.
\end{abstract}

\footnotetext{
* Autor para correspondencia.

Correo electrónico: horacioinvestigacion@hotmail.com (H. Márquez-González).
} 


\section{KEYWORDS}

Congenital cardiac disease;

Echocardiogram; Magnetic resonance imaging
Conclusiones: La vena contracta y el porcentaje VC/AP son variables que tienen alta correlación con la fracción y el volumen regurgitante de la resonancia magnética nuclear para evaluar la insuficiencia pulmonar en pacientes en posoperatorio de cardiopatía congénita.

(c) 2015 Sociedad Colombiana de Cardiología y Cirugía Cardiovascular. Publicado por Elsevier España, S.L.U. Este es un artículo Open Access bajo la licencia CC BY-NC-ND (http:// creativecommons.org/s/by-nc-nd/4.0/).

\section{Correlation between magnetic resonance and transthoracic echocardiogram for the assessment of pulmonary insufficiency in pediatric patients with congenital heart disease}

\begin{abstract}
Motivation: To establish the correlation and concordance of the degree of severity of pulmonary insufficiency between echocardiography and magnetic resonance in pediatric population of a third level hospital.

Methods: 20 patients diagnosed with congenital heart disease and with pulmonary insufficiency were studied, they underwent a transthoracic echocardiogram and a magnetic resonance. The relationship of the vena contracta, the pulmonary regurgitation jet, the pressure half-time time, the presence of reversed end-diastolic flow and the pulmonary insufficiency index were calculated. With regards to the magnetic resonance, both fraction and regurgitant volume were measured. An analysis of the correlation was conducted.by means of the Spearman correlation and the Tau B.

Results: Corrected Fallot's tetralogy represented $60 \%$ of the sample. The correlation was significant in the fraction and the regurgitant volume of the magnetic resonance, as well as in the echocardiographic variables of $M$-mode pulmonary insufficiency, vena contracta and vena contracta/pulmonary artery percentage. The correlation between the degrees of pulmonary insufficiency by magnetic resonance and echocardiogram was $0.85(\mathrm{p}<0.001)$.

Conclusions: Vena contracta and the VC/PA percentage are variables that present high correlation with the fraction and the regurgitant volume of nuclear magnetic resonance to assess pulmonary insufficiency in patients after a congenital cardiac disease surgery.

(c) 2015 Sociedad Colombiana de Cardiología y Cirugía Cardiovascular. Published by Elsevier España, S.L.U. This is an open access article under the CC BY-NC-ND license (http:// creativecommons.org/licenses/by-nc-nd/4.0/).
\end{abstract}

\section{Introducción}

La corrección de las cardiopatías congénitas que afectan al ventrículo derecho, habitualmente incluyen en sus procedimientos plastia de la válvula pulmonar que condiciona a futuro la disfunción del aparato valvular ${ }^{1-3}$. En su mayoría, la insuficiencia pulmonar es bien tolerada en los primeros años, no obstante la historia natural condiciona el incremento de la severidad que más tarde se asociará con intolerancia al ejercicio, disfunción ventricular, arritmia e incluso muerte $^{4,5}$. Lo anterior obliga a los cardiólogos pediatras a mantener un seguimiento estrecho que les permita identificar el momento oportuno para realizar alguna maniobra quirúrgica paliativa a esta complicación.

El ecocardiograma transtorácico (ETT) es un estudio no invasivo que tiene una alta sensibilidad para su diagnóstico; sin embargo, la resonancia magnética nuclear (RMN) es el estándar de oro para evaluar la fracción regurgitante (FR) y la función del $\mathrm{VD}^{6}$, pero con desventajas como elevados costos y contraindicación para realizarse en pacientes con marcapasos y algunas otras estructuras metálicas.

Así mismo, la RMN puede clasificar la gravedad de la insuficiencia pulmonar; existe poca evidencia entre la correlación de esta herramienta con otras más accesibles en este reducido grupo de pacientes que amerita seguimiento continuo.

\section{Métodos}

Con el objetivo de establecer la correlación de la severidad de la insuficiencia pulmonar entre el ETT y la RMN en pacientes pediátricos con cardiopatía congénitas, se realizó un estudio transversal, analítico y prospectivo en el área de Cardiología pediátrica del Centro Médico Nacional 20 de Noviembre del ISSSTE (Instituto de Seguridad y Servicios Sociales de los Trabajadores del Estado).

La población diana fueron enfermos atendidos en la consulta de Cardiología pediátrica con el diagnóstico de cardiopatías congénitas, entre el año 2011 y 2012 . Se incluyeron pacientes cardiópatas posoperados de cirugía correctiva de patología congénita (corrección total con parche en hamaca, tipo Rastelli, valvuloplastia percutánea o ampliación valvular) con insuficiencia pulmonar secundaria, en clase funcional । y II clasificada por los criterios de la New York Hearth Association (NYHA) modificada por Ross $^{7}$ con evolución estable en el último año. Se excluyeron aquellos 
con cirugía correctiva en el último año, ajuste farmacológico o adición de algún fármaco nuevo por motivo de descompensación hemodinámica, y se eliminaron aquellos que presentaron deterioro del estado general (clase funcional, descompensación hemodinámica, infecciones graves o internamiento) entre ambos estudios.

A los sujetos seleccionados se les realizó RMN y ETT en un periodo menor de 90 días entre ambas pruebas.

El ETT fue realizado por cardiólogo pediatra ecocardiografista con kappa $>0,9$ intra e interobservador. Las mediciones se hicieron de acuerdo con las guías de la Sociedad Americana de Ecocardiografía para el análisis del ventrículo derecho ${ }^{8}$. El ecocardiógrafo utilizado fue Philips iE33 y el transductor de 5 y $7 \mathrm{~Hz}$. Se midieron los siguientes parámetros:

- Excursión sistólica del anillo tricuspídeo (TAPSE, su sigla en inglés, por tricuspid anular plane systolic excursion $)^{9}$ : se calculó la distancia de la excursión sistólica del segmento anular del ventrículo derecho a lo largo de su plano longitudinal, a partir de una ventana apical de 4 cámaras medido en milímetros.

- Índice de desempeño miocárdico (Tei) ${ }^{10}$ : en la proyección apical cuatro cámaras, calculado a partir de la relación entre el tiempo isovolumétrico y el tiempo eyectivo o (TRIV + TCIV)/TE; la unidad es en $\mathrm{cm} / \mathrm{s}$.

- Relación entre vena contracta (VC) del chorro de insuficiencia en relación con el anillo pulmonar $(\% \mathrm{VC})^{11}$ : mediante la proyección eje corto paraesternal, se midió el anillo de la arteria pulmonar y posteriormente el jet de regurgitación de la insuficiencia pulmonar y se realizó la ecuación para determinar el porcentaje de la vena contracta respecto al anillo valvular pulmonar. Como marcador de insuficiencia pulmonar grave se seleccionó una anchura del jet mayor o igual del $50 \%$ del diámetro anular.

- Tiempo de hemipresión $\left(\mathrm{T}^{1 / 2}\right)^{12}$ : se determinó utilizando el modo doppler (pulsado y continuo) sobre el jet de insuficiencia pulmonar; se calculó la desaceleración de la pendiente en la velocidad del jet regurgitante en milisegundos.

- Presencia o ausencia de flujo diastólico reverso en tronco pulmonar: mediante el eje paraesternal corto en modo bidimensional se ubicó el doppler pulsado sobre el jet de regurgitación pulmonar guiado por el doppler color (no existe unidad de medición, solo ausencia o presencia).

- Índice de insuficiencia pulmonar (IIP) por modo $\mathrm{M}^{13}$ : en el eje paraesternal en modo bidimensional se localizó la aorta y la rama pulmonar derecha (RPD) y se congeló la imagen para efectuar dos medidas: diámetro en sístole y en diástole; se realizó la ecuación (diámetro diastólico de la RPD en mm/diámetro sistólico de la RPD en $\mathrm{mm}$ ) y el resultado se clasificó en grados de gravedad de acuerdo con el rango: ligera $(1,18-1,20)$, moderada $(1,21-1,39)$ y grave $\geq 1,40$.

Todos los estudios de RMN se hicieron con equipo 1,5-T Philips y 3-T Siemens y se evaluaron en el software cardiaco ARGUS. Los niños menores de 4 años fueron sedados y la adquisición se llevó a cabo mientras el paciente respiró espontáneamente. En niños mayores no se usó sedación y la adquisición se hizo durante el final de la espiración. Los estudios fueron posprocesados en una estación satélite y fueron analizados por un cardiólogo especialista en imagen.

Se realizaron secuencias de mapeo de flujo mediante cortes axiales en la válvula pulmonar para calcular la fracción regurgitante (FR) con el programa ARGUS y se clasificó en gravedad con base en el porcentaje: leve FR (15-24\%), moderada (25-39\%) y grave ( $\geq 40 \%)$.

La selección de los pacientes de interés fue por muestreo por conveniencia de casos consecutivos en el periodo comprendido.

El protocolo fue aprobado por el comité local de ética en investigación del Hospital sede del estudio.

\section{Análisis estadístico}

En la estadística descriptiva se emplearon medidas de tendencia central (mediana) y dispersión (rangos intercuartiles) para las variables cuantitativas, mientras que las frecuencias y porcentajes se utilizaron para las variables cualitativas.

La estadística inferencial empleada fue la prueba de correlación de Spearman para cálculo de $r^{2}$ entre los resultados del ETT y la fracción regurgitante y el volumen regurgitante de la arteria pulmonar de la RM.

La concordancia entre el grado de severidad de la insuficiencia pulmonar por RM y ETT se hizo con prueba de Tau B. Su usó el programa estadístico SPSS versión 18 para Windows.

\section{Resultados}

Se obtuvo un total de 20 pacientes, 11 (55\%) mujeres, con mediana de edad de 12 años (6-14) y mediana de peso de $35 \mathrm{~kg}$ (19-49). Según la RMN, 10 (50\%) presentaron insuficiencia pulmonar severa, 6 (30\%) moderada y el resto ligera (tabla 1). La tetralogía de Fallot corregida por cirugía tipo Rastelli, fue la causa de la insuficiencia pulmonar en 12 (60\%) pacientes (fig. 1).

Se encontró una correlación estadísticamente significativa (tabla 2) de la fracción regurgitante de la arteria pulmonar con la vena contracta $\left(r^{2}=0,8, p<0,001\right)$ y el porcentaje VC/AP $\left(r^{2}=0,8, p<0,001\right)$, y en el VR de la arteria pulmonar con insuficiencia pulmonar en modo $M$ $\left(r^{2}=0,85, p=0,01\right)$, porcentaje VC/AP $\left(r^{2}=0,6, p=0,02\right)$ y TAPSE $\left(r^{2}=0,7, p=0,001\right)$.

La mayor concordancia se presentó entre los grados de insuficiencia pulmonar de la RMN con los grados de insuficiencia pulmonar por VC en el ETT (tabla 3).

\section{Discusión}

Los pacientes en condición de posoperatorio con insuficiencia pulmonar residual, deberían tener seguimiento con RMN, lo cual no siempre es posible debido a que esta tecnología y los recursos humanos que la interpretan no son asequibles en todos los centros hospitalarios. En este estudio se documenta la correlación del ETT como principal herramienta para evaluar y clasificar el grado de severidad de la insuficiencia pulmonar en pacientes con corrección quirurgica o intervencionista. 
Tabla 1 Características generales de la población

\begin{tabular}{|c|c|c|}
\hline Variable & Frecuencia/mediana & Porcentaje (\%)/rango intercuartil (25-75\%) \\
\hline \multicolumn{3}{|l|}{ Sexo } \\
\hline Masculino & 9 & $45 \%$ \\
\hline Femenino & 11 & $55 \%$ \\
\hline Edad (años) & 10 & $6-14$ \\
\hline Peso (kg) & 33 & $19-49$ \\
\hline \multicolumn{3}{|l|}{ Insuficiencia pulmonar por RMN } \\
\hline Ligera & 4 & $20 \%$ \\
\hline Moderada & 6 & $30 \%$ \\
\hline Severa & 10 & $50 \%$ \\
\hline \multicolumn{3}{|l|}{ Resonancia magnética } \\
\hline Fracción regurgitante (\%) & 38 & $30-42$ \\
\hline Volumen regurgitante $(\mathrm{ml})$ & 18 & $14-46$ \\
\hline \multicolumn{3}{|l|}{ Variables ecocardiográficas } \\
\hline ITV onda S (cm) & 35 & $13-45$ \\
\hline TEI (\%) & 0,37 & $0,31-0,50$ \\
\hline $\mathrm{T} 1 / 2 \mathrm{P}(\mathrm{m} / \mathrm{s})$ & 87 & $72-106$ \\
\hline VTD (mL) & 61 & $38-96$ \\
\hline VTS (mL) & 20 & $8-45$ \\
\hline FE VD(\%) & 46 & $38-50$ \\
\hline VC $(\mathrm{mm})$ & 9 & $8-10$ \\
\hline $\mathrm{IT}(\mathrm{mm} \mathrm{Hg})$ & 20 & $10-30$ \\
\hline$\% \mathrm{VC} / \mathrm{AP}$ & 36 & $31-51$ \\
\hline TAPSE $(\mathrm{mm})$ & 15 & $14-18$ \\
\hline
\end{tabular}

$\mathrm{RMN}=$ resonancia magnética nuclear, TEI= índice de Tei, $\mathrm{T} 1 / 2 \mathrm{P}=$ tiempo de hemipresión de la arteria pulmonar, VTD= volumen telediastólico, VTS= volumen telesistólico, FE VD= fracción de expulsión del ventrículo derecho, VC= vena contracta, \% VC/AP= porcentaje vena contracta/arteria pulmonar, TAPSE= tiempo de excursión del anillo tricuspídeo.

Los resultados de este trabajo demostraron correlación y concordancia estadísticamente significativa entre la vena contracta y el porcentaje VC/AP con la fracción y el volumen regurgitante de la RMN. Al momento de

Procedimientos realizados en los casos de insuficiencia pulmonar

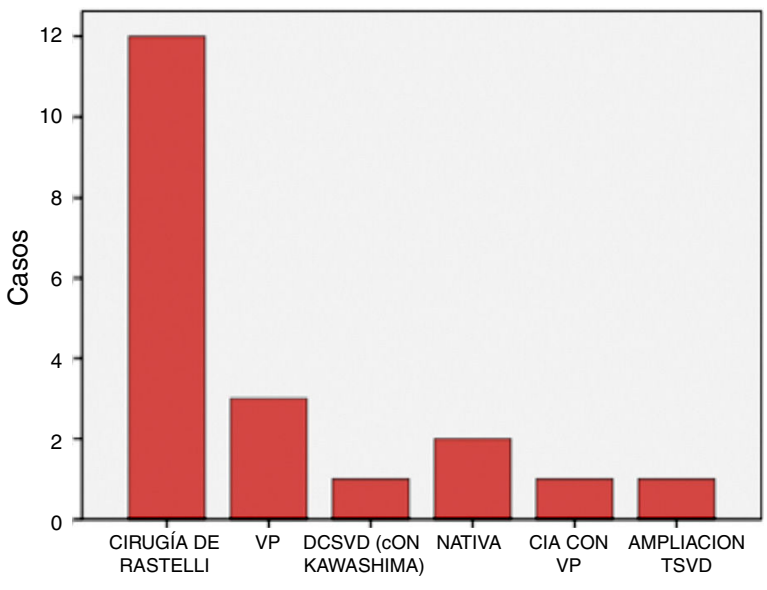

VP = valvuloplastía pulmonar, DSCVD = doble cámara de salida de ventrículo derecho, $\mathrm{CIA}$ con VP = defecto interatrial con valvuloplastía pulmonar, Ampliación TSVD = ampliación del tracto de salida del ventrículo derecho.

Figura 1 Se grafican las cardiopatías congénitas y las causas de insuficiencia pulmonar de los pacientes que integraron el estudio. estratificar el grado de severidad, dicha significancia se mantuvo.

La Sociedad Americana de Ecocardiografía sugiere que la vena contracta y el porcentaje de la misma en relación con el diámetro de la arteria pulmonar, son una forma confiable de predecir la insuficiencia pulmonar severa. Lo anterior concuerda con lo publicado por Renella et al. ${ }^{14}$ quienes encontraron en 36 casos con insuficiencia pulmonar severa documentada por RMN, que el ancho del chorro de regurgitación pulmonar $\geq 50 \%$ es un buen predictor de insuficiencia pulmonar grave, con OR 36; IC95\% 3,8-342, $\mathrm{p}=0,002$ ).

Por su parte, Mercer-Rosa et al. integraron una cohorte de 143 pacientes con corrección de tetralogía de Fallot en la que calcularon la validez de la vena contracta y el porcentaje VC/AP y consideraron la RMN como estándar de oro con una sensibilidad de $97 \%$ y una tasa de $36 \%$ de falsos positivos cuando la fracción regurgitante por RMN era menor al $20 \%{ }^{15}$.

El IIP por modo M mide la variación del diámetro sistólicodiastólico en la rama pulmonar derecha mediante eco 2D por modo $M$, lo cual se basa en el hecho de que al analizar las secuencias de cine en RMN se observan cambios en el diámetro de las ramas pulmonares asociados con insuficiencia pulmonar; eventualmente se utiliza la rama derecha por la facilidad para visualizarla mediante eje supraesternal. Desde el punto de vista práctico, se señala que los valores $\geq 1,18, \geq 1,21 \mathrm{y} \geq 1,41$ muestran correlación con los valores por $\mathrm{RMN} \geq 15 \%$, $\geq 25 \%$ y $>40 \%$, establecido así por la Sociedad Americana de Ecocardiografía y evaluación de 
Tabla 2 Prueba de correlación entre la fracción regurgitante y el volumen regurgitante de la insuficiencia pulmonar por RMN con las variables ecocardiográficas en ventana transtorácica

\begin{tabular}{|c|c|c|c|}
\hline \multicolumn{2}{|c|}{ Variables ecocardiográficas } & \multirow{2}{*}{$\begin{array}{l}\text { Fracción regurgitante por RMN } \\
(\%)\end{array}$} & \multirow{2}{*}{$\begin{array}{l}\text { Volumen regurgitante por } \mathrm{RMN}(\mathrm{ml}) \\
0,7\end{array}$} \\
\hline ITV de la onda S & $r^{2}$ & & \\
\hline$(\mathrm{cm})$ & $\mathrm{p}$ & 0,6 & 0,01 \\
\hline \multirow[t]{2}{*}{ TEI (\%) } & $r^{2}$ & 0,1 & $-0,05$ \\
\hline & $\mathrm{p}$ & 0,1 & 0,8 \\
\hline \multirow[t]{2}{*}{$\mathrm{T} 1 / 2 \mathrm{P}(\mathrm{m} / \mathrm{s})$} & $r^{2}$ & $-0,18$ & 0,05 \\
\hline & $\mathrm{p}$ & 0,1 & 0,7 \\
\hline \multirow[t]{2}{*}{ VTD (ml) } & $r^{2}$ & 0,1 & 0,56 \\
\hline & $\mathrm{p}$ & 0,6 & 0,02 \\
\hline \multirow[t]{2}{*}{ VTS (ml) } & $r^{2}$ & 0,18 & 0,68 \\
\hline & $\mathrm{p}$ & 0,1 & 0,001 \\
\hline \multirow[t]{2}{*}{ FE VD (\%) } & $r^{2}$ & $-0,1$ & $-0,48$ \\
\hline & $\mathrm{p}$ & 0,6 & 0,05 \\
\hline \multirow[t]{2}{*}{$\mathrm{VC}(\mathrm{mm})$} & $r^{2}$ & 0,8 & 0,17 \\
\hline & $\mathrm{p}$ & 0,01 & 0,5 \\
\hline \multirow[t]{2}{*}{ IT (mm Hg) } & $r^{2}$ & 0,3 & 0,1 \\
\hline & $\mathrm{p}$ & 0.6 & 0,4 \\
\hline \multirow[t]{2}{*}{$\% \mathrm{VC} / \mathrm{AP}$} & $r^{2}$ & 0,8 & 0,6 \\
\hline & $\mathrm{p}$ & $<0,001$ & 0,02 \\
\hline TAPSE & $r^{2}$ & $-0,2$ & 0,7 \\
\hline$(\mathrm{mm})$ & $\mathrm{p}$ & 0,3 & 0,001 \\
\hline
\end{tabular}

TEI= índice de Tei, $\mathrm{T} 1 / 2 \mathrm{P}=$ tiempo de hemipresión de la arteria pulmonar, VTD= volumen telediastólico, VTS= volumen telesistólico, FE $\mathrm{VD}=$ fracción de expulsión del ventrículo derecho, $\mathrm{VC}=$ vena contracta, \% VC/AP= porcentaje vena contracta/arteria pulmonar, TAPSE= tiempo de excursión del anillo tricuspídeo.

Tabla 3 Concordancia entre la severidad de la insuficiencia pulmonar medida por ETT y RMN

\begin{tabular}{llcl}
\hline \multicolumn{1}{c}{ Variable } & \multicolumn{1}{c}{$\begin{array}{l}\text { Insuficiencia pulmonar } \\
\text { por \% VC/AP ETT }\end{array}$} & $\begin{array}{l}\text { Insuficiencia pulmonar por } \\
\text { ETT en modo bidimensional }\end{array}$ \\
\hline $\begin{array}{l}\text { Insuficiencia pulmonar por grados (leve, moderado, grave) } \\
\text { Por resonancia magnética }\end{array}$ & t & 0,85 & 0,46 \\
\hline
\end{tabular}

Grados de \% VC/AP: $<0,20=$ ligera, 0,21-0,49= moderada, $>0,50=$ severa.

Grados de insuficiencia pulmonar en modo $M=$ ligera $(1,18-1,20)$, moderada $(1,21-1,39)$ y grave $\geq 1,40$.

enfermedad valvular cardiaca por resonancia magnética en 2002. Festa et al. en pacientes con corrección de tetralogía de Fallot, relacionaron la fracción regurgitante por RMN mayor al 25\% con el IIP, y documentaron una correlación $r^{2}=0,85$ en población menor de 18 años con un área bajo la curva (AUC) de 0,99 (IC95\% 0,95-0,99) ${ }^{16}$. Cuando se comparó con una fracción regurgitante por RMN mayor de $40 \%$, el AUC fue de 0,92 (IC95\%, 0,82-95). No obstante, Renella et al. ${ }^{14}$ reportaron que el índice de insuficiencia pulmonar por modo $M$ no alcanza significancia estadística como predictor independiente de insuficiencia pulmonar ni severa ni grave con OR 1,9 (IC95\% 0,5-7; $p=33)$. Cabe mencionar que solo utilizo un valor de índice de insuficiencia pulmonar por modo $M$, siendo este un diámetro $<0,77$, el cual, de acuerdo con los rangos que se proponen, quedaría clasificado como insuficiencia pulmonar leve. En el estudio el resultado fue una correlación positiva estadísticamente significativa con $r^{2}=0,50, p=0,05$.

Con base en los resultados de este estudio puede inferirse que el ETT continúa siendo una herramienta útil, reproducible, accesible y no invasiva en el seguimiento de los pacientes pediátricos con insuficiencia pulmonar. Estos parámetros son reproducibles con facilidad en el consultorio u hospital y las modalidades son básicas en casi cualquier equipo ecocardiográfico. De ninguna manera se pretende excluir la información aportada por la RMN, que continúa siendo el elemento estándar para el estudio del ventrículo derecho.

La principal limitante en este trabajo es el reducido tamaño de muestra, que si bien permite obtener resultados similares a los de otros autores, impide formular otro tipo de conclusiones. Además pueden presentarse los sesgos característicos de todo estudio transversal.

Los resultados medidos entre la RMN y el ETT son altamente correlacionados y justifican la realización de estudios con mayor número de sujetos para realizar un protocolo de prueba diagnóstica.

\section{Conclusiones}

La vena contracta y el porcentaje VC/AP son variables que tienen alta correlación con la fracción y el volumen 
regurgitante de la RMN para evaluar la insuficiencia pulmonar en pacientes en condición de posoperados de cardiopatía congénita.

\section{Responsabilidades éticas}

Protección de personas y animales. Los autores declaran que para esta investigación no se han realizado experimentos en seres humanos ni en animales.

Confidencialidad de los datos. Los autores declaran que en este artículo no aparecen datos de pacientes.

Derecho a la privacidad y consentimiento informado. Los autores declaran que en este artículo no aparecen datos de pacientes.

\section{Conflicto de intereses}

Los autores declaran no tener ningún conflicto de interés.

\section{Bibliografía}

1. Parekh DR. A review of heart failure in adults with congenital heart disease. Methodist Debakey Cardiovasc J. 2011;7(2):26-32.

2. Norozi K, Wessel A, Alpers V, Arnhold JO, Geyer S, Zoege M, Buchhorn R. Incidence and risk distribution of heart failure in adolescents and adults with congenital heart disease after cardiac surgery. Am J Cardiol. 2006;97:1238-43.

3. Rendón I, Soto M, Jaramillo M, Palacio AC, Restrepo JA. Tetralogía de Fallot y embarazo. Rev Colomb Cardiol. 2014;21: 246-50.

4. Saleh S, Liakopoulos OJ, Buckberg GD. The septal motor of biventricular function. Eur J Cardio-thorac Surg. 2006;295:S126-38.

5. Chturvedi RR, Redington AN. Pulmonary regurgitation in congenital heart disease. Heart. 2007;93:880-9.

6. Davlouros PA, Kilner PJ, Hornung TS, Li W, Francis JM, Moon JC, et al. Right ventricular function in adults with repaired tetralogy of Fallot assessed with cardiovascular magnetic resonance imaging: detrimental role of right ventricular outflow aneurysm or akinesia and adverse right-to-left ventricular interaction. J Am Coll Cardiol. 2002;40:2044-52.

7. Rosenthal D, Christsant MR, Edens E, Mahony L, Canter C, Colan $S$, et al. International Society for Heart and Lung transplantation: practice guidelines for management of heart failure in children. J Heart Lung Transplant. 2004;23:1313-33.

8. Rudiski L, Wyman C, Afilalo J, Hua L, Handschumacher M, Chandresekaran K, et al. Guidelines for echocardiographic assessment of the right heart in adults: A report from the American Society of Echochardiography. J Am Soc Echocardiogr. 2010;23, 865-713.

9. Núñez-Gil I, Rubio MD, Cartón AJ, López-Romero P, Deiros L, García-Guereta L, et al. Determinación de valores normalizados del desplazamiento sistólico del plano del anillo tricuspídeo (TAPSE) en 405 niños y adolescentes españoles. Rev Esp Cardiol. 2011;64:674-80.

10. Tei $C$. New non-invasive index for combined systolic and diastolic ventricular function. J. Cardiol. 1995;26:135-6.

11. Mele D, Vandervoort P, Palacios I. Proximal jet size by Doppler color flow mapping predicts severity of mitral regurgitation. Circulation. 1995;91:746-54.

12. Feigenbaum H. Echocardiographic examination of the left ventricle. Circulation. 1975;51:1.

13. Ferreiro M, Toro L, Andia M, Zelada P, Alcántara A, Castillo $M E$, et al. Estudio preliminar de índices ecocardiográficos que permitan optimizar la indicación de resonancia cardíaca en pacientes con tetralogía de allot reparada. Rev Chil Cardiol. 2013;32(1):46-50.

14. Renella P, Aboulhosn J, Lohan D, Jonnala P, Finn P, Satou Gary, Williams R, Child J. Two-Dimensional and Doppler echocardiography realiably predict severe pulmonary regurgitation as quantified by cardiac magnetic resonance. J Am Soc Echocardiogr. 2010;23:880-6.

15. Mercer-Rosa L, Yang W, Kutty S, Rychik J, Fogel M, Goldmuntz E. Quantifyng pulmonary regurgitation an right ventricular function in surgically repaired tetralogy of Fallot: a comparative analysis of echocardiography and magnetic resonance imaging. Circ Cardiovasc Imaging. 2012;5:637-43.

16. Festa P, Ait-Ali L, Minichilli F, Kristo I, Deiana M, Picano E. A new simple method to estimate pulmonary regurgitation by echocardiography in operated Fallot:Comparison with magnetic resonance imaging and performance test evaluation. J Am Soc Echocardiogr. 2010;23:496-503. 